\title{
On $\left(\alpha^{*}, \psi\right)$-contractive multi-valued mappings
}

\author{
Muhammad Usman Ali and Tayyab Kamran²*
}

\section{"Correspondence:}

tayyabkamran@gmail.com

2 Department of Mathematics,

Quaid-i-Azam University, Islamabad,

Pakistan

Full list of author information is

available at the end of the article

\begin{abstract}
In this paper, we generalize the contractive condition for multi-valued mappings given by Asl, Rezapour and Shahzad in 2012. We establish some fixed point theorems for multi-valued mappings from a complete metric space to the space of closed or bounded subsets of the metric space satisfying generalized $\left(\alpha^{*}, \psi\right)$-contractive condition.
\end{abstract}

MSC: $47 \mathrm{H} 10 ; 54 \mathrm{H} 25$

Keywords: $\alpha$-admissible; $\alpha^{*}$ - $\psi$-contractive mapping; generalized $\left(\alpha^{*}, \psi\right)$-contractive mapping

\section{Introduction}

Samet et al. [1] introduced the notion of $\alpha$ - $\psi$-contractive self-mappings of a metric space. Recently, Asl et al. [2] introduced the notion of $\alpha^{*}-\psi$-contractive mappings to extend the notion $\alpha-\psi$-contractive mappings. In this paper, we generalize the notion of $\alpha^{*}-\psi$ contractive mappings and prove some fixed point theorems for such mappings.

Let $\Psi$ be a family of nondecreasing functions, $\psi:[0, \infty) \rightarrow[0, \infty)$ such that $\sum_{n=1}^{\infty} \psi^{n}(t)<\infty$ for each $t>0$, where $\psi^{n}$ is the $n$th iterate of $\psi$. It is known that for each $\psi \in \Psi$, we have $\psi(t)<t$ for all $t>0$ and $\psi(0)=0$ for $t=0$ [1]. Let $(X, d)$ be a metric space. A mapping $G: X \rightarrow X$ is called $\alpha-\psi$-contractive if there exist two functions $\alpha: X \times X \rightarrow[0, \infty)$ and $\psi \in \Psi$ such that $\alpha(x, y) d(G x, G y) \leq \psi(d(x, y))$ for each $x, y \in X$. A mapping $G: X \rightarrow X$ is called $\alpha$-admissible [1] if $\alpha(x, y) \geq 1 \Rightarrow \alpha(G x, G y) \geq 1$. We denote by $N(X)$ the space of all nonempty subsets of $X$, by $B(X)$ the space of all nonempty bounded subsets of $X$ and by $C L(X)$ the space of all nonempty closed subsets of $X$. For $A \in N(X)$ and $x \in X, d(x, A)=\inf \{d(x, a): a \in A\}$. For every $A, B \in B(X)$, $\delta(A, B)=\sup \{d(a, b): a \in A, b \in B\}$. When $A=\{x\}$, we denote $\delta(A, B)$ by $\delta(x, B)$. For every $A, B \in C L(X)$, let

$$
H(A, B)= \begin{cases}\max \left\{\sup _{x \in A} d(x, B), \sup _{y \in B} d(y, A)\right\} & \text { if the maximum exists; } \\ \infty & \text { otherwise. }\end{cases}
$$

Such a map $H$ is called generalized Hausdorff metric induced by $d$. Let $(X, \preceq, d)$ be an ordered metric space and $A, B \subseteq X$. We say that $A \prec_{r} B$ if for each $a \in A$ and $b \in B$, we have $a \preceq b$. We give a few definitions and the result due to Asl et al. [2] for convenience.

(c) 2013 Ali and Kamran; licensee Springer. This is an Open Access article distributed under the terms of the Creative Commons Attribution License (http://creativecommons.org/licenses/by/2.0), which permits unrestricted use, distribution, and reproduction in any medium, provided the original work is properly cited. 
Definition 1.1 [2] Let $(X, d)$ be a metric space and let $\alpha: X \times X \rightarrow[0, \infty)$ be a mapping. A mapping $G: X \rightarrow C L(X)$ is $\alpha^{*}$-admissible if $\alpha(x, y) \geq 1 \Rightarrow \alpha^{*}(G x, G y) \geq 1$, where $\alpha^{*}(G x, G y)=\inf \{\alpha(a, b): a \in G x, b \in G y\}$.

Definition 1.2 [2] Let $(X, d)$ be a metric space. A mapping $G: X \rightarrow C L(X)$ is called $\alpha^{*}-\psi$ contractive if there exist two functions $\alpha: X \times X \rightarrow[0, \infty)$ and $\psi \in \Psi$ such that

$$
\alpha^{*}(G x, G y) H(G x, G y) \leq \psi(d(x, y))
$$

for all $x, y \in X$.

Theorem 1.3 [2] Let $(X, d)$ be a complete metric space, let $\alpha: X \times X \rightarrow[0, \infty)$ be a function, let $\psi \in \Psi$ be a strictly increasing map and $T$ be a closed-valued, $\alpha^{*}$-admissible and $\alpha^{*}$ - $\psi$-contractive multi-function on $X$. Suppose that there exist $x_{0} \in X$ and $x_{1} \in G x_{0}$ such that $\alpha\left(x_{0}, x_{1}\right) \geq 1$. Assume that if $\left\{x_{n}\right\}$ is a sequence in $X$ such that $\alpha\left(x_{n}, x_{n+1}\right) \geq 1$ for all $n$ and $x_{n} \rightarrow x$, then $\alpha\left(x_{n}, x\right) \geq 1$ for all $n$. Then $G$ has a fixed point.

\section{Main results}

We begin this section by introducing the following definition.

Definition 2.1 Let $(X, d)$ be a metric space and let $G: X \rightarrow C L(X)$ be a mapping. We say that $G$ is generalized $\left(\alpha^{*}, \psi\right)$-contractive if there exists $\psi \in \Psi$ such that

$$
\alpha^{*}(G x, G y) d(y, G y) \leq \psi(d(x, y))
$$

for each $x \in X$ and $y \in G x$, where $\alpha^{*}(G x, G y)=\inf \{\alpha(a, b): a \in G x, b \in G y\}$.

Note that an $\alpha^{*}-\psi$-contractive mapping is generalized $\left(\alpha^{*}, \psi\right)$-contractive. In case when $\psi \in \Psi$ is strictly increasing, generalized $\left(\alpha^{*}, \psi\right)$-contractive is called strictly generalized $\left(\alpha^{*}, \psi\right)$-contractive. The following lemma is inspired by [3, Lemma 2.2].

Lemma 2.2 Let $(X, d)$ be a metric space and $B \in C L(X)$. Then, for each $x \in X$ with $d(x, B)>$ 0 and $q>1$, there exists an element $b \in B$ such that

$$
d(x, b)<q d(x, B) .
$$

Proof It is given that $d(x, B)>0$. Choose

$$
\epsilon=(q-1) d(x, B) .
$$

Then, by using the definition of $d(x, B)$, it follows that there exists $b \in B$ such that

$$
d(x, b)<d(x, B)+\epsilon=q d(x, B) .
$$

Lemma 2.3 Let $(X, d)$ be a metric space and $G: X \rightarrow C L(X)$. Assume that there exists a sequence $\left\{x_{n}\right\}$ in $X$ such that $\lim _{n \rightarrow \infty} d\left(x_{n}, G x_{n}\right)=0$ and $x_{n} \rightarrow x \in X$. Then $x$ is a fixed point of $G$ if and only if the function $f(\xi)=d(\xi, G \xi)$ is lower semi-continuous at $x$. 
Proof Suppose $f(\xi)=d(\xi, G \xi)$ is lower semi-continuous at $x$, then

$$
d(x, G x) \leq \liminf _{n} f\left(x_{n}\right)=\liminf _{n} d\left(x_{n}, G x_{n}\right)=0 .
$$

By the closedness of $G$ it follows that $x \in G x$. Conversely, suppose that $x$ is a fixed point of $G$, then $f(x)=0 \leq \liminf _{n} f\left(x_{n}\right)$.

Theorem 2.4 Let $(X, d)$ be a complete metric space and let $G: X \rightarrow C L(X)$ be an $\alpha^{*}$-admissible strictly generalized $\left(\alpha^{*}, \psi\right)$-contractive mapping. Assume that there exist $x_{0} \in X$ and $x_{1} \in G x_{0}$ such that $\alpha\left(x_{0}, x_{1}\right) \geq 1$. Then $x$ is a fixed point of $G$ if and only if $f(\xi)=d(\xi, G \xi)$ is lower semi-continuous at $x$.

Proof By the hypothesis, there exist $x_{0} \in X$ and $x_{1} \in G x_{0}$ such that $\alpha\left(x_{0}, x_{1}\right) \geq 1$. If $x_{0}=x_{1}$, then we have nothing to prove. Let $x_{0} \neq x_{1}$. If $x_{1} \in G x_{1}$, then $x_{1}$ is a fixed point. Let $x_{1} \notin G x_{1}$. Since $G$ is $\alpha^{*}$-admissible, so $\alpha^{*}\left(G x_{0}, G x_{1}\right) \geq 1$, we have

$$
0<d\left(x_{1}, G x_{1}\right) \leq \alpha^{*}\left(G x_{0}, G x_{1}\right) d\left(x_{1}, G x_{1}\right) .
$$

For given $q>1$ by Lemma 2.2, there exists $x_{2} \in G x_{1}$ such that

$$
0<d\left(x_{1}, x_{2}\right)<q d\left(x_{1}, G x_{1}\right) .
$$

It follows from (2.3), (2.4) and (2.1) that

$$
0<d\left(x_{1}, x_{2}\right)<q \psi\left(d\left(x_{0}, x_{1}\right)\right) .
$$

It is clear that $x_{1} \neq x_{2}$ and $\alpha\left(x_{1}, x_{2}\right) \geq 1$. Thus $\alpha^{*}\left(G x_{1}, G x_{2}\right) \geq 1$. Since $\psi$ is strictly increasing, by (2.5), we have

$$
\psi\left(d\left(x_{1}, x_{2}\right)\right)<\psi\left(q \psi\left(d\left(x_{0}, x_{1}\right)\right)\right) .
$$

Put $q_{1}=\frac{\psi\left(q \psi\left(d\left(x_{0}, x_{1}\right)\right)\right)}{\psi\left(d\left(x_{1}, x_{2}\right)\right)}$, then $q_{1}>1$. If $x_{2} \in G x_{2}$, then $x_{2}$ is a fixed point. Let $x_{2} \notin G x_{2}$, then by Lemma 2.2 , there exists $x_{3} \in G x_{2}$ such that

$$
\begin{aligned}
0 & <d\left(x_{2}, x_{3}\right)<q_{1} d\left(x_{2}, G x_{2}\right) \leq q_{1} \alpha^{*}\left(G x_{1}, G x_{2}\right) d\left(x_{2}, G x_{2}\right) \\
& \leq q_{1} \psi\left(d\left(x_{1}, x_{2}\right)\right)=\psi\left(q \psi\left(d\left(x_{0}, x_{1}\right)\right)\right) .
\end{aligned}
$$

It is clear that $x_{2} \neq x_{3}, \alpha\left(x_{2}, x_{3}\right) \geq 1$ and $\psi\left(d\left(x_{2}, x_{3}\right)\right)<\psi^{2}\left(q \psi\left(d\left(x_{0}, x_{1}\right)\right)\right)$. Now put $q_{2}=$ $\frac{\psi^{2}\left(q \psi\left(d\left(x_{0}, x_{1}\right)\right)\right)}{\psi\left(d\left(x_{2}, x_{3}\right)\right)}$. Then $q_{2}>1$. If $x_{3} \in G x_{3}$, then $x_{3}$ is a fixed point. Let $x_{3} \notin G x_{3}$. Then by Lemma 2.2 there exists $x_{4} \in G x_{3}$ such that

$$
\begin{aligned}
0 & <d\left(x_{3}, x_{4}\right)<q_{2} d\left(x_{3}, G x_{3}\right) \leq q_{2} \alpha^{*}\left(G x_{2}, G x_{3}\right) d\left(x_{3}, G x_{3}\right) \\
& \leq q_{2} \psi\left(d\left(x_{2}, x_{3}\right)\right)=\psi^{2}\left(q \psi\left(d\left(x_{0}, x_{1}\right)\right)\right) .
\end{aligned}
$$

By continuing the same process, we get a sequence $\left\{x_{n}\right\}$ in $X$ such that $x_{n+1} \in G x_{n}$. Also, $x_{n} \neq x_{n+1}, \alpha\left(x_{n}, x_{n+1}\right) \geq 1$ and $0<d\left(x_{n}, x_{n+1}\right)<\psi^{n-1}\left(q \psi\left(d\left(x_{0}, x_{1}\right)\right)\right)$ or

$$
0<d\left(x_{n}, G x_{n}\right)<\psi^{n-1}\left(q \psi\left(d\left(x_{0}, x_{1}\right)\right)\right) .
$$


For each $m>n$, we have

$$
d\left(x_{n}, x_{m}\right) \leq \sum_{i=n}^{m-1} d\left(x_{i}, x_{i+1}\right)<\sum_{i=n}^{m-1} \psi^{i-1}\left(q \psi\left(d\left(x_{0}, x_{1}\right)\right)\right)
$$

Since $\psi \in \Psi$, it follows that $\left\{x_{n}\right\}$ is a Cauchy sequence in $X$. Thus there is $x \in X$ such that $x_{n} \rightarrow x$. Letting $n \rightarrow \infty$ in (2.6), we have

$$
\lim _{n \rightarrow \infty} d\left(x_{n}, G x_{n}\right)=0
$$

The rest of the proof follows from Lemma 2.3.

Example 2.5 Let $X=\mathbb{R}$ be endowed with the usual metric $d$. Define $G: X \rightarrow C L(X)$ and $\alpha: X \times X \rightarrow[0, \infty)$ by

$$
G x= \begin{cases}{[x, \infty)} & \text { if } x \geq 0, \\ \left(-\infty,-x^{2}\right] & \text { if } x<0\end{cases}
$$

and

$$
\alpha(x, y)= \begin{cases}1 & \text { if } x, y \geq 0 \\ 0 & \text { otherwise }\end{cases}
$$

Let $\psi(t)=\frac{t}{2}$ for all $t \geq 0$. For each $x \in X$ and $y \in G x$, we have

$$
\alpha^{*}(G x, G y) d(y, G y)=0 \leq \frac{1}{2} d(x, y) .
$$

Hence $G$ is a strictly generalized $\left(\alpha^{*}, \psi\right)$-contractive mapping. Clearly, $G$ is $\alpha^{*}$-admissible. Also, we have $x_{0}=1$ and $x_{1}=1 \in G x_{0}$ such that $\alpha\left(x_{0}, x_{1}\right)=1$. Therefore, all conditions of Theorem 2.4 are satisfied and $G$ has infinitely many fixed points. Note that Theorem 1.3 in Section 1 is not applicable here. For example, take $x=1$ and $y=-1$.

Corollary 2.6 Let $(X, \preceq, d)$ be a complete ordered metric space, $\psi \in \Psi$ be a strictly increasing map and $G: X \rightarrow C L(X)$ be a mapping such that for each $x \in X$ and $y \in G x$ with $x \preceq y$, we have

$$
d(y, G y) \leq \psi(d(x, y)) .
$$

Also, assume that

(i) there exist $x_{0} \in X$ and $x_{1} \in G x_{0}$ such that $x_{0} \preceq x_{1}$,

(ii) if $x \preceq y$, then $G x \prec_{r} G y$.

Then $x$ is a fixed point of $G$ if and only if $f(\xi)=d(\xi, G \xi)$ is lower semi-continuous at $x$.

Proof Define $\alpha: X \times X \rightarrow[0, \infty)$ by

$$
\alpha(x, y)= \begin{cases}1 & \text { if } x \preceq y \\ 0 & \text { otherwise }\end{cases}
$$


By using condition (i) and the definition of $\alpha$, we have $\alpha\left(x_{0}, x_{1}\right)=1$. Also, from condition (ii), we have $x \preceq y$ implies $G x \prec_{r} G y$; by using the definitions of $\alpha$ and $\prec_{r}$, we have $\alpha(x, y)=1$ implies $\alpha^{*}(G x, G y)=1$. Moreover, it is easy to check that $G$ is a strictly generalized $\left(\alpha^{*}, \psi\right)$ contractive mapping. Therefore, by Theorem 2.4, $x$ is a fixed point of $G$ if and only if $f(\xi)=d(\xi, G \xi)$ is lower semi-continuous at $x$.

Definition 2.7 Let $(X, d)$ be a metric space and $G: X \rightarrow B(X)$ be a mapping. We say that $G$ is a generalized $\left(\alpha^{*}, \psi, \delta\right)$-contractive mapping if there exists $\psi \in \Psi$ such that

$$
\alpha^{*}(G x, G y) \delta(y, G y) \leq \psi(d(x, y))
$$

for each $x \in X$ and $y \in G x$, where $\alpha^{*}(G x, G y)=\inf \{\alpha(a, b): a \in G x, b \in G y\}$.

Lemma 2.8 Let $(X, d)$ be a metric space and $G: X \rightarrow B(X)$. Assume that there exists a sequence $\left\{x_{n}\right\}$ in $X$ such that $\lim _{n \rightarrow \infty} \delta\left(x_{n}, G x_{n}\right)=0$ and $x_{n} \rightarrow x \in X$. Then $\{x\}=G x$ if and only if the function $f(\xi)=\delta(\xi, G \xi)$ is lower semi-continuous at $x$.

Proof Suppose that $f(\xi)=\delta(\xi, G \xi)$ is lower semi-continuous at $x$, then

$$
\delta(x, G x) \leq \liminf _{n} f\left(x_{n}\right)=\liminf _{n} \delta\left(x_{n}, G x_{n}\right)=0 .
$$

Hence, $\{x\}=G x$ because $\delta(A, B)=0$ implies $A=B=\{a\}$. Conversely, suppose that $\{x\}=$ $G x$. Then $f(x)=0 \leq \liminf _{n} f\left(x_{n}\right)$.

Theorem 2.9 Let $(X, d)$ be a complete metric space and let $G: X \rightarrow B(X)$ be an $\alpha^{*}$-admissible generalized $\left(\alpha^{*}, \psi, \delta\right)$-contractive mapping. Assume that there exist $x_{0} \in X$ and $x_{1} \in G x_{0}$ such that $\alpha\left(x_{0}, x_{1}\right) \geq 1$. Then there exists $x \in X$ such that $\{x\}=G x$ if and only iff $f(\xi)=\delta(\xi, G \xi)$ is lower semi-continuous at $x$.

Proof By the hypothesis of the theorem, there exist $x_{0} \in X$ and $x_{1} \in G x_{0}$ such that $\alpha\left(x_{0}, x_{1}\right) \geq 1$. Assume that $x_{0} \neq x_{1}$, for otherwise, $x_{0}$ is a fixed point. Let $x_{1} \notin G x_{1}$. As $G$ is $\alpha^{*}$-admissible, we have $\alpha^{*}\left(G x_{0}, G x_{1}\right) \geq 1$. Then

$$
\delta\left(x_{1}, G x_{1}\right) \leq \alpha^{*}\left(G x_{0}, G x_{1}\right) \delta\left(x_{1}, G x_{1}\right) \leq \psi\left(d\left(x_{0}, x_{1}\right)\right) .
$$

Since $G x_{1} \neq \emptyset$, there is $x_{2} \in G x_{1}$. Then

$$
0<d\left(x_{1}, x_{2}\right) \leq \delta\left(x_{1}, G x_{1}\right)
$$

From (2.12) and (2.13), we have

$$
0<d\left(x_{1}, x_{2}\right) \leq \psi\left(d\left(x_{0}, x_{1}\right)\right)
$$

Since $\psi$ is nondecreasing, we have

$$
\psi\left(d\left(x_{1}, x_{2}\right)\right) \leq \psi^{2}\left(d\left(x_{0}, x_{1}\right)\right) .
$$


As $x_{2} \in G x_{1}$, we have $\alpha\left(x_{1}, x_{2}\right) \geq 1$. Since $G x_{2} \neq \emptyset$, there is $x_{3} \in G x_{2}$. Assume that $x_{2} \neq x_{3}$, for otherwise, $x_{2}$ is a fixed point of $G$. Then

$$
\begin{aligned}
0<d\left(x_{2}, x_{3}\right) & \leq \delta\left(x_{2}, G x_{2}\right) \leq \alpha^{*}\left(G x_{1}, G x_{2}\right) \delta\left(x_{2}, G x_{2}\right) \\
& \leq \psi\left(d\left(x_{1}, x_{2}\right)\right) \leq \psi^{2}\left(d\left(x_{0}, x_{1}\right)\right) .
\end{aligned}
$$

Since $\psi$ is nondecreasing, we have

$$
\psi\left(d\left(x_{2}, x_{3}\right)\right) \leq \psi^{3}\left(d\left(x_{0}, x_{1}\right)\right)
$$

By continuing in this way, we get a sequence $\left\{x_{n}\right\}$ in $X$ such that $x_{n+1} \in G x_{n}$ and $x_{n} \neq x_{n+1}$ for $n=0,1,2,3, \ldots$. Further we have

$$
0<d\left(x_{n}, x_{n+1}\right) \leq \delta\left(x_{n}, G x_{n}\right) \leq \psi^{n}\left(d\left(x_{0}, x_{1}\right)\right) .
$$

For each $m>n$, we have

$$
d\left(x_{n}, x_{m}\right) \leq \sum_{i=n}^{m-1} d\left(x_{i}, x_{i+1}\right) \leq \sum_{i=n}^{m-1} \psi^{i}\left(d\left(x_{0}, x_{1}\right)\right) .
$$

Since $\psi \in \Psi$, it follows that $\left\{x_{n}\right\}$ is a Cauchy sequence in $X$. As $X$ is complete, there exists $x \in X$ such that $x_{n} \rightarrow x$. Letting $n \rightarrow \infty$ in (2.18), we have

$$
\lim _{n \rightarrow \infty} \delta\left(x_{n}, G x_{n}\right)=0
$$

The rest of the proof follows from Lemma 2.8 .

Example 2.10 Let $X=\{0,2,4,6,8,10, \ldots\}$ be endowed with the usual metric $d$. Define $G: X \rightarrow B(X)$ and $\alpha: X \times X \rightarrow[0, \infty)$ by

$$
G x= \begin{cases}\{(x-2), x\} & \text { if } x \neq 0, \\ \{0\} & \text { if } x=0\end{cases}
$$

and

$$
\alpha(x, y)= \begin{cases}0 & \text { if } x=y \neq 0 \\ 1 & \text { if } x=y=0 \\ \frac{1}{4} & \text { otherwise }\end{cases}
$$

Let $\psi(t)=\frac{t}{2}$ for all $t \geq 0$. For each $x \in X$ and $y \in G x$, we have

$$
\alpha^{*}(G x, G y) \delta(y, G y) \leq \frac{1}{2}(d(x, y)) .
$$

Hence $G$ is a generalized $\left(\alpha^{*}, \psi, \delta\right)$-contractive mapping. Clearly, $G$ is $\alpha^{*}$-admissible. Also, we have $x_{0}=0 \in X$ and $x_{1}=0 \in G 0$ such that $\alpha\left(x_{0}, x_{1}\right)=1$. Therefore, all conditions of Theorem 2.9 are satisfied and $G$ has infinitely many fixed points. 
Corollary 2.11 Let $(X, \preceq, d)$ be a complete ordered metric space, $\psi \in \Psi$ and $G: X \rightarrow B(X)$ be a mapping such that for each $x \in X$ and $y \in G x$ with $x \preceq y$, we have

$$
\delta(y, G y) \leq \psi(d(x, y))
$$

\section{Also, assume that}

(i) there exists $x_{0} \in X$ such that $\left\{x_{0}\right\} \prec_{1} G x_{0}$, i.e., there exists $x_{1} \in G x_{0}$ such that $x_{0} \preceq x_{1}$,

(ii) if $x \preceq y$, then $G x \prec_{r} G y$.

Then there exists $x \in X$ such that $\{x\}=G x$ if and only if $f(\xi)=\delta(\xi, G \xi)$ is lower semicontinuous at $x$.

Proof Define $\alpha: X \times X \rightarrow[0, \infty)$ by

$$
\alpha(x, y)= \begin{cases}1 & \text { if } x \preceq y \\ 0 & \text { otherwise }\end{cases}
$$

By using condition (i) and the definition of $\alpha$, we have $\alpha\left(x_{0}, x_{1}\right)=1$. Also, from condition (ii), we have $x \preceq y$ implies $G x \prec_{r} G y$, by using the definitions of $\alpha$ and $\prec_{r}$, we have $\alpha(x, y)=1$ implies $\alpha^{*}(G x, G y)=1$. Moreover, it is easy to check that $G$ is a generalized $\left(\alpha^{*}, \psi, \delta\right)$ contractive mapping. Therefore, by Theorem 2.9, there exists $x \in X$ such that $\{x\}=G x$ if and only if $f(\xi)=\delta(\xi, G \xi)$ is lower semi-continuous at $x$.

\section{Competing interests}

The authors declare that they have no competing interests.

\section{Authors' contributions}

Both authors contributed equally in this article.

\section{Author details}

${ }^{1}$ Centre for Advanced Mathematics and Physics, National University of Sciences and Technology H-12, Islamabad,

Pakistan. ${ }^{2}$ Department of Mathematics, Quaid-i-Azam University, Islamabad, Pakistan.

\section{Acknowledgements}

Authors are grateful to referees for their suggestions and careful reading.

Received: 14 February 2013 Accepted: 10 May 2013 Published: 28 May 2013

\section{References}

1. Samet, B, Vetro, C, Vetro, P: Fixed point theorems for $\alpha$ - $\psi$-contractive type mappings. Nonlinear Anal. 75, 2154-2165 (2012)

2. Asl, JH, Rezapour, S, Shahzad, N: On fixed points of $\alpha$ - $\psi$-contractive multifunctions. Fixed Point Theory Appl. 2012, 212 (2012). doi:10.1186/1687-1812-2012-212

3. Kamran, T: Mizoguchi-Takahashi's type fixed point theorem. Comput. Math. Appl. 57, 507-511 (2009)

doi:10.1186/1687-1812-2013-137

Cite this article as: Ali and Kamran: On $\left(\alpha^{*}, \psi\right)$-contractive multi-valued mappings. Fixed Point Theory and Applications $20132013: 137$ 